\title{
Afrontamiento familiar en pacientes con Covid 19
}

\author{
Family coping in patients with Covid 19
}

Afrontamiento familiar

\begin{abstract}
Margoth Elizabeth Villegas Chiriboga Mg. ${ }^{1}$
Roberth Patricio Zambrano Ubillus Dr. ${ }^{2}$

Henry Gilberto Villegas Sánchez Dr. ${ }^{3}$
\end{abstract}

${ }^{1}$ Universidad Estatal del Sur de Manabí, margoth.villegas@unesum.edu.ec, ORCID ID: 0003-4637-5396.

${ }^{2}$ Instituto Superior Universitario Portoviejo, roberthzamubi@ hotmail.com

${ }^{3}$ Ministerio de Salud Pública 09D21, henry.villegas@gmail.com

Contacto: margoth.villegas@unesum.edu.ec

Recibido: 25-04-2020

Aprobado: 23-06-2020

\section{Resumen}

El COVID-19 ha provocado el aislamiento a nivel mundial producto del periodo de cuarentena recomendado por la Organización Mundial de la Salud lo cual ha conllevado a familiares de pacientes a enfrentar este acontecimiento estresante, convirtiéndose en una problemática social, para hacer frente al confinamiento y las repercusiones que puede afectar en la familia es necesario adoptar ciertas estrategias de afrontamiento familiar. Los Objetivos son dar respuesta a las estrategias de afrontamiento para los familiares que estén contagiados con la Covid-19, describir complicaciones, medidas y fitofármacos a utilizar para tratar la enfermedad y las afecciones que causa Métodos se realizó una revisión de la literatura científica de carácter multidisciplinario-cualitativo y descriptivo, considerando la base de datos de carácter nacional e internacional, como son; Medline, Cochrane, BVS, Lilacs, Pubmed, publicaciones de América Latina, América del sur y las publicaciones de la OMS y OPS. Resultados. La búsqueda permitió seleccionar 24 artículos, cuya información es analizada con el fin de aportar al conocimiento sobre las estrategias de afrontamiento a adoptar en la pandemia para mitigar efectos y secuelas en la salud familiar, las personas se ven obligadas a relacionarse constantemente de manera familiar y personal, lo que puede generar ciertas situaciones problemáticas como disputas, y malentendidos.
Conclusiones. Las estrategias de apoyo físico, psicológico, anímico, los hábitos, creencias espirituales, y la comunicación constante combaten la soledad, el miedo disminuye niveles de estrés y depresión, conservando un ambiente positivo y adecuado en el paciente, familia y la sociedad.

Palabras clave: Estrategias de afrontamiento, miedo, ansiedad, confinamiento social, estabilidad psicológica.

\section{Summary}

COVID-19 has caused isolation worldwide as a result of the quarantine period recommended by the World Health Organization, which has led patients' relatives to face this stressful event, becoming a social problem, to face confinement and the repercussions that it can affect in the family it is necessary to adopt certain family coping strategies. The Objectives are to respond to the coping strategies for family members who are infected with Covid-19, describe complications, measures and phytopharmaceuticals to be used to treat the disease and the conditions it causes. multidisciplinary-qualitative and descriptive, considering the national and international database, as they are; Medline, Cochrane, BVS, Lilacs, Pubmed, Latin American publications, South America and WHO and PAHO publications. Results. The search allowed to select 24 articles, whose information is analyzed in order to contribute to the knowledge about the coping strategies to adopt in the pandemic to mitigate effects and sequelae on family health, 
HIGIA DE LA SALUD

people are forced to constantly interact in a family and personal, which can generate certain problematic situations such as disputes, and misunderstandings. Conclusions. The strategies of physical, psychological, emotional support, habits, spiritual beliefs, and constant communication combat loneliness, fear reduces levels of stress and depression, preserving a positive and adequate environment in the patient, family and society.

Keywords: Coping strategies, fear, anxiety, social confinement, psychological stability.

\section{Introducción}

El afrontamiento familiar es la capacidad de la familia para enfrentarse, movilizarse y poner en acción medidas que actúen sobre las exigencias que demandan cambios ante la aparición de acontecimientos estresantes, las personas que eligen estrategias adecuadas o saludables de afrontamiento muestran un mejor estado emocional e inmunológico, provocado por el alivio del estrés y expresado mediante la conducta, aun así es necesario que uno de los integrantes de la familia tome el rol de líder para guiar a los demás

En la actualidad, estudios detallan que el alarmante número de casos confirmados de COVID-19 a nivel global, es una realidad preocupante y desalentadora en el mundo, llena de impotencia a las diferentes masas poblacionales, por el hecho de desconocer como eliminar radicalmente o disminuir rápidamente esta pandemia, aunado a las secuelas económicas y sociales, como la cuarentena y el aislamiento social de las familias, sobre todo en personas vulnerables, ya que han aumentado la ansiedad, depresión, soledad, insomnio e incluso tristeza, desesperación y suicidio, es por ello, que es muy importante conocer y aplicar las estrategias de afrontamiento, que han servido de manera efectiva para el autocuidado y disminuir los efectos negativos en la salud mental (1).

La investigación de esta problemática social se realizó con el fin de dar conocer a la sociedad la importancia de las estrategias aplicadas en los pacientes con la Covid-19, la recuperación de un familiar y cómo sobrellevarlo en épocas de confinamiento, qué daño psicosocial puede causar al tener un familiar contagiado y medidas a tomar para llevar esta situación.

Los principales objetivos son dar respuesta a las estrategias de afrontamiento para los familiares que estén contagiados con la Covid-19, además describir complicaciones, medidas y fitofármacos a utilizar para tratar la enfermedad y las afecciones que causa, el fin del proyecto se centró en concientizar, motivar y orientar a la familia sobre a qué recursos recurrir para su debida protección, sobre todo a que no rompan la comunicación con sus familiares a pesar de que
Periodo. Enero - Junio 2020

Vol. 2, Nro. 1, Publicado 2020-06-30 se encuentren contagiados por la Covid-19, es una investigación con un enfoque cualitativo en donde se utilizaron herramientas y técnicas, además se realizó un video educativo con el que se motivó a las personas a no aislarse del cariño de sus familiares.

Los contenidos se desarrollaron de manera detalla fundamentados en evidencias, se indagó la manera en que repercute el afrontamiento en pacientes con la Covid-19, desde fuentes confiables como la OMS y artículos científicos, se aplicaron métodos de observación que permite analizar la situación por la que están atravesando las personas que padecen covid-19, de la frustración, necesidades y falta de información que vive la gente día a día al lidiar con desconocimiento esta pandemia y sus familiares contagiados, surge el tema de la investigación.

La salud familiar ha constituido una preocupación para todos aquellos que trabajan con la familia (2) y los altos niveles de estrés familiar pueden conducir a la elección de estrategias de afrontamiento poco saludables, generar un entorno inseguro y un mayor riesgo de resultados negativos en personas vulnerables, (3) por ello las estrategias de afrontamiento familiar surgen en medio de la pandemia por la covid-19, dentro de ella los índices de muerte y el deterioro en nuestro sistema inmune una vez superada, siendo una de sus causas, el déficit de conocimiento sobre estrategias para el afrontamiento de la enfermedad.

Dejan como consecuencia las muertes de familias completas debido a la novedad que implica un virus que es nuevo relativamente en cuanto a síntomas, complicaciones, mecanismo de transmisión y las diferentes patologías que causa, por lo que se establece una revisión bibliográfica para describir cada variable.

La investigación clínica con plantas medicinales chinas contra el COVID-19 presenta resultados alentadores en pacientes con cuadros leves y moderados, pero también en casos severos, (4) por esto las formas de combatirla, las maneras de afrontarlas y asimilarlas, se remiten a conocimientos empíricos en donde las personas usan medicinas caseras sin conocer los fitofármacos correctos usados para combatir esta enfermedad y se evidencia como resultado que esta crisis cada vez se vuelve más grave, porque empiezan a padecer nuevos síntomas y complicaciones por la ingesta de fitofármacos que no le ayudan sino que deterioran su salud.

Los familiares identifican la necesidad de intimidad como fundamental en el proceso de morir, aquellos que no han podido vivirlo así, lo expresan como un trauma (5). Por lo que la imposibilidad de comunicarse con sus familiares, de poder verlos y visitarlos, conduce a 
HIGIA DE LA SALUD

situaciones de estrés y depresión por el temor a contagiarlos y sobre todo la posibilidad de que quizá no puedan volverlos a ver a causa de la muerte en caso de que no superen la enfermedad. Materiales y métodos:

Se realizó una investigación bibliográfica de tipo descriptiva y explicativa, en donde a partir de las referencias encontradas se analizaron las causas del problema por lo que se desarrollaron las estrategias de afrontamiento familiar en pacientes infectados por la Covid-19.

Esta investigación tiene un enfoque cualitativo el cual permitió desarrollar el tema, interpretar los fenómenos, hasta lograr profundizar el estudio relacionado con el afrontamiento familiar.

Se utilizó como técnica un vídeo educativo, la herramienta empleada fue la observación de comentarios y reacciones sobre el mismo para poder realizar un análisis minucioso.

\section{Resultados:}

Las teorías y modelos están cada vez más presentes en los programas formativos de enfermería, sobre todo a nivel de licenciatura, especialidad, maestrías y doctorados, pues las teorías constituyen un conjunto organizado de conocimientos abstractos, avalados por la investigación científica y el análisis lógico, que proporcionan las bases para comprender la realidad en enfermería, y capacitan al enfermero para comprender el por qué un hecho ocurre. (6) Es por esto importante resaltar que las teorías de enfermería son la base a partir de la cual se puede describir y explicar las diferentes situaciones que están sucediendo y que a pesar de estar escritas con anterioridad se encuentran vigente sin importar la transición de los años, ya que a través de ella se evidencian diferentes procesos por los que atraviesa el ser humano a pesar de su evolución, por lo que las siguientes teorías que se van abordar constituyen a factores determinantes dentro de la investigación a pesar de ser un virus nuevo.

Es interesante analizar la Teoría del afrontamiento, estrés y procesos cognitivos de Lazarus y Folkman. Esta teoría forma parte de los denominados modelos transaccionales del estrés, ya que tiene en cuenta cómo interactúa la persona ante un ambiente y una situación concreta, considerando la influencia de sus evaluaciones y cogniciones, según Lazarus, una situación resulta estresante a raíz de las transacciones entre persona y entorno, mismas que dependen del impacto del estresor ambiental, a su vez, dicho impacto está mediatizado por dos variables las evaluaciones que hace la persona del agente estresor los recursos personales, sociales o culturales de que dispone la persona al enfrentarse a tal agente. (7) La teoría del afrontamiento, estrés y procesos cognitivos de Lazarus y Folkman, es centrada en
Periodo. Enero - Junio 2020

Vol. 2, Nro. 1, Publicado 2020-06-30

establecer el afrontamiento que se debe hacer ante una situación estresante, depende del contexto, pero sobre todo que el afrontamiento familiar se vuelve una necesidad al superar las diferentes situaciones que se presentan para poder salir adelante con la enfermedad y las reacciones adversas como la muerte, es vital que se entienda la importancia de esta teoría que nos da a comprender todo lo que puede presentarse siendo capaz de enfrentarlo.

Se considera importante la Teoría del Autocuidado de Dorothea Orem en la adopción de estrategias de afrontamiento por parte del ser humano.

La teoría de Dorothea explica el concepto de auto cuidado como una contribución constante del individuo a su propia existencia: "el auto cuidado es una actividad aprendida por los individuos, orientada hacia un objetivo, es una conducta que existe en situaciones concretas de la vida, dirigida por las personas sobre sí mismas, hacia los demás o hacia el entorno, para regular los factores que afectan a su propio desarrollo y funcionamiento en beneficio de su vida, salud o bienestar.

La pandemia de coronavirus (covid-19) ha alterado la vida familiar en todo el mundo. Debido a las características y normas impuestas por el estado de alarma y el confinamiento en casa, las personas se ven obligadas a relacionarse constantemente de manera familiar y personal, lo que puede generar ciertas situaciones problemáticas y aumentar las probabilidades de que aparezcan disputas, conflictos, discusiones y malentendidos. Sin embargo, algunas personas lidian con algún familiar enfermo, lo que desencadena pensamientos negativos relacionado con la ansiedad por las limitaciones de estar en contacto personal, comunicarse plenamente, y desconocer acerca de este virus (8).

En estudios de impacto psicológico en la población general de China el $84,7 \%$ de sujetos que participaron permanecían en casa entre 2024 horas. Además un 75,2\% estaban preocupados por la posibilidad de que un miembro de su familia se infecte con la Covid-19 y un $75,1 \%$ estaban satisfechos por la cantidad de información de salud disponible (9). No obstante, se percibió que las estrategias como, controlar las emociones negativas, el apoyo familiar o de amigos, ejercicios físicos, meditación en conjunto con la información necesaria y positiva ayudan a conllevar este problema y es fundamental para reducir este impacto de la enfermedad (10).

La destrucción de hogares, separación de la escuela, la familia y los amigos pueden crear una gran cantidad de estrés y ansiedad para los niños, el impacto emocional de la emergencia en los 
HIGÍA DE LA SALUD niños depende de las características, experiencias del niño, circunstancias sociales y económicas de

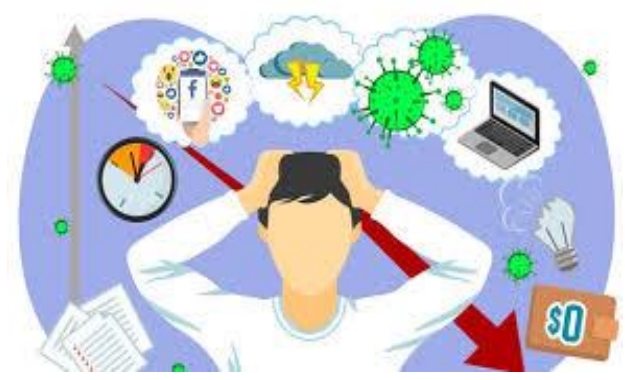

la familia, la comunidad, y la disponibilidad de recursos locales. (11)

A falta de vacunas contra el Covid-19, muchos ecuatorianos han acudido a métodos ingeniosos, la mayoría criticados por autoridades, con los que esperan derrotar al coronavirus, un mal que en casi tres meses ha dejado más de 36.700 contagiados y 3.100 muertos en el país. En todos los tiempos, el tratamiento de enfermedades con plantas medicinales ha sido una práctica muy común en las comunidades rurales, práctica que se mantiene hasta la actualidad en el Ecuador y en muchos países del mundo como el eucalipto se usa para las afecciones respiratorias y el bulbo del ajo se usa para reforzar el sistema inmunitario y ayuda a prevenir infecciones, o a superarlas con más rapidez. Como lo evidencian numerosos estudios en países europeos, como España, Alemania o Francia, una de cada tres personas usa este tipo de tratamiento (12).

Pueden sentir que debido a las preocupaciones y pocas estrategias de afrontamiento, tienen un pensamiento negativo sobre las consecuencias del COVID 19, en casos que no puede dormir, o sienta sueño diurno, por otro lado puede también sentir con el aislamiento, si es el caso de la tendencia a dormir en exceso, cambiar cronobiológicamente los ritmos de sueño y vigilia a través de siestas o periodos de sueño que no son los usuales dentro de los hábitos que cotidianamente tenía antes del problema (13).

En medio de la incertidumbre los pacientes y familias acuden a la medicina alternativa como son los fitofármacos, sabiendo que son productos obtenidos a través de diferentes procesos, empleando exclusivamente materias primas vegetales, tienen una finalidad profiláctica, curativa, paliativa o para fines de diagnóstico, también son medicamentos que contienen como principio activo exclusivamente para plantas, ya sea sus partes de plantas, ingredientes vegetales o preparaciones obtenidas a partir de ellas.

Son fármacos que contienen, como sustancias activas, preparaciones de partes vegetales en una forma galénica específica y que forman parte de una terapia medicamentosa racional en el sentido
Periodo. Enero - Junio 2020

Vol. 2, Nro. 1, Publicado 2020-06-30

de la medicina científica, además se emplean para el tratamiento de enfermedades 0 padecimientos definidos. (14)

Estrategias de afrontamiento familiar ante la

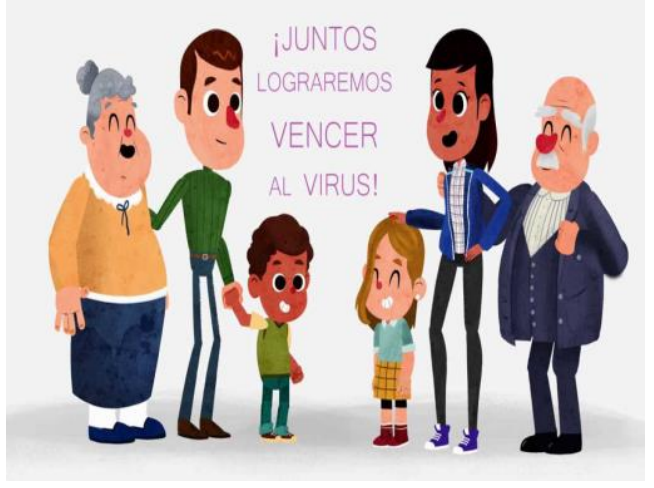

pandemia por Covid-19 - UNICEF

1.-Hay que identificar los canales oficiales y confiables de información sobre el COVID 19. De este modo, se requiere evitar la saturación de noticias sobre el virus o la desinformación; ya que ambos son contraindicados. De ahí se sugiere revisar las noticias a través de fuentes oficiales en momentos específicos del día y evitar lecturas permanentes, sobre todo en redes sociales.

2.- Construir participativamente con la familia una serie de estrategias, para la provisión de alimentos y medicinas, ajustándose a las condiciones y restricciones de circulación. No comprar en exceso, hacer una lista de las necesidades del hogar para comprar lo necesario evitando las compras de pánico.

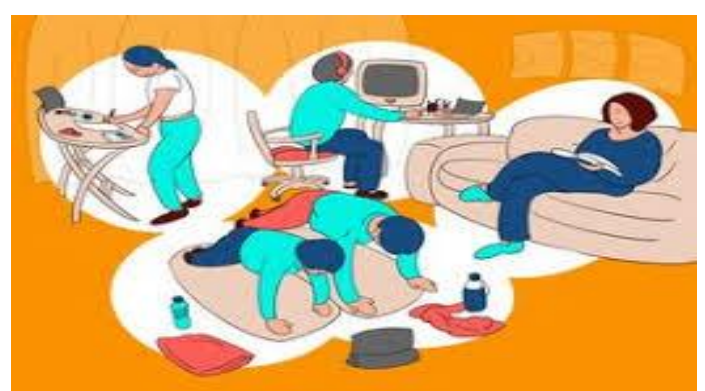

3.-Crear rutinas en el hogar, adaptar las rutinas diarias de la familia para organizar el teletrabajo y las tareas de cuidado de nuestros familiares, en especial de niños, niñas adolescentes, adultos mayores o personas que requieran atención especial. En lo posible, designar responsabilidades entre los miembros de la familia, reconocer la importancia de cada persona en la familia.

4.- Asignar lugares en la casa para el teletrabajo y el estudio, así como para compartir momentos de esparcimiento y diversión con la familia. Es importante encontrar actividades que promuevan la alegría y el disfrute

5.- Permitir que los miembros de la familia expresen sus sentimientos y temores con amplitud, brindándoles respuestas 
HIGIA DE LA SALUD

tranquilizadoras y objetivas. Recuérdeles que hacer lo que piden las autoridades de salud a toda la población es una importante contribución (15). 6.- Evitar pensamientos catastrofistas a futuro, pues esto puede generar angustia. Sabemos que estamos en un momento complejo pero que pasará. Intentar hacer una lista de planes positivos y de nuevos aprendizajes para cuando la emergencia sanitaria termine.

7. Mantener comunicación con la familia y los compañeros de trabajo ayuda a disminuir la sensación de aislamiento. Recuerde que el humor es un gran recurso para bajar la ansiedad y afrontar las situaciones difíciles.

\section{Conclusiones:}

$>$ El SARS-CoV-2 es el virus causante de la enfermedad de la Covid-19, se transmite de manera directa e indirecta por medio de goticulas procedentes de una persona infectada. Los síntomas característicos son la anosmia y ageusia. Afecta principalmente al sistema respiratorio presentando complicaciones graves como la neumonía, infarto y la muerte.

$>$ Los fitofármacos son usados como medicina alternativa ya que estos contienen como principio activo extractos de plantas medicinales beneficioso para aliviar la sintomatología que produce este virus
Periodo. Enero - Junio 2020 Vol. 2, Nro. 1, Publicado 2020-06-30 durante los primeros 5 días antes de presentar complicaciones.

$>$ Las familias que aplican las estrategias de afrontamiento tales como combatir la soledad, el miedo y evitar la sobreexposición a la información durante la pandemia, ven disminuido sus niveles de estrés y depresión frente a quienes no las aplican, en donde gracias a estas estrategias mantienen un ambiente positivo y adecuado.

$>$ Se realizó un video educativo compartido a través del canal de YouTube "Pedagogía del Cuidado" dirigido a la comunidad con el fin de dar a conocer diferentes estrategias acordes a la edad para afrontar de manera positiva el confinamiento, enfatizando la importancia de la salud mental dentro de la familia.

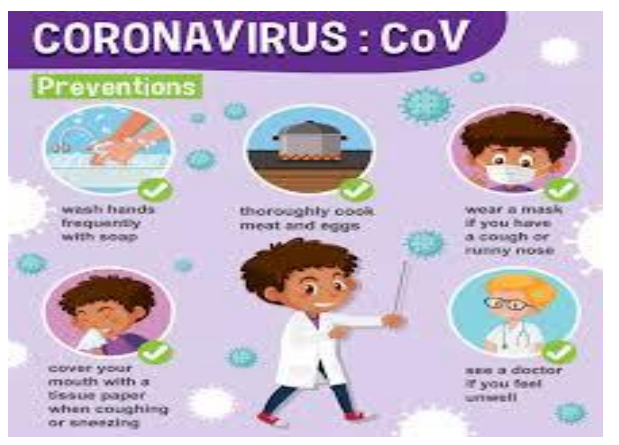

\section{Colaboradores en la Investigación:}

Andrade Zambrano Simón, Arriaga Ruiz Carlos, Bowen Cevallos Christian, Burgos Burgos Melissa, Briones Alcívar Romario, Cedeño Marcillo Erick, Cedeño Solórzano Gabriel, Cevallos Alvia Marina, Constantine Chávez Anahí.

Estudiantes de la Carrera de Enfermería de la Universidad Estatal del Sur de Manabí

\section{Bibliografía}

1. Nereida Valero C MVCÁDMMTP. revistas.uta.edu.ec. [Online].; 2020 [cited 202009 09. Available from: blob:https://web.whatsapp.com/4e75d6d9-2542-4bf7-9f9c-e1b88d6961f4.

2. José Manuel Martínez-Montilla BAM,MDGM. Scielo. [Online].; 2017 [cited 202008 25. Available from: http://scielo.isciii.es/scielo.php?pid=S1695-61412017000300576\&script=sci_arttext\&tlng=pt

3. Carmen Orte Socías LBByLNC. [Online]. [cited 202008 28. Available from: file:///C:/Users/LENOVO/Downloads/297-Preprint\%20Text-337-1-10-20200502.pdf.

4. Enrique Moncada-Mapelli ASG. Medicina tradicional y COVID-19, oportunidad para la revaloración de las Plantas Medicinales Peruanas. [Online].; 2020 [cited 20200825.

5. Begoña García MAS. Abordaje del duelo y de la muerte en familiares de pacientes con COVID-19: revisión narrativa. [Online].; 2020 [cited $2020 \quad 08$ 25. Available from: https://www.sciencedirect.com/science/article/pii/S1130862120303089.

6. Román CAL. Revista Cubana de enfermería. [Online].; 2016 [cited 20200907. Available from: http://revenfermeria.sld.cu/index.php/enf/article/view/1587/301.

7. Ruiz L. [Online]. [cited 202008 13. Available from: https://psicologiaymente.com/psicologia/teoriaestres- 
lazarus\#: :text=Lazarus\%20(1966)\%2C\%20tambi\%C3\%A9n\%20desarrollada,del\%20contexto\%20y $\% 20$ otras\%20variables.

8. Georgieva M. [Online]. [cited 2020 Septiembre 8. Available from: https://www.psicologiamadrid.es/blog/articulos/psicologia-y-coronavirus/consejos-para-convivir-enfamilia-ante-en-confinamiento-en-casa-por-covid-19.

9. Lozano-Vargas A. Neuro-Psiquiatría. [Online].; 2020 [cited 2020 Septiembre 8. Available from: http://www.scielo.org.pe/scielo.php?pid=S0034-85972020000100051\&script=sci_arttext.

10 Activa Psicología y Formación Logo. Activa Psicología y Formación Logo. [Online]. [cited 2020

Septiembre 8. Available from: http://www.activapsicologia.com/familias-ingresados-covid/.

11 SALUD OMDL. ORGANIZACION MUNDIAL DE LA SALUD. [Online].; 2020 [cited 2020

- AGOSTO $25 . \quad$ Available from:
https://www.who.int/docs/defaultsource/coronaviruse/mentalhealthconsiderations.pdf?sfvrsn=6d3578 af_10.

12 Efe. CORONAVIRUS ECUADOR. [Online].; 2020 [cited 202009 08. Available from:

. https://www.efe.com/efe/america/sociedad/a-falta-de-vacunas-los-ecuatorianos-acuden-metodosingeniosos-para-combatir-el-covid-19/20000013-4254922.

13 Briones A. Mantener su distancia para mantenerse seguro. [Online].; 2020 [cited 2020 septiembre 03.

- Available from: https://www.salud.gob.ec/wp-content/uploads/2020/03/lineamientosCOVID19_DNCSS_31032020-ECU-911.pdf.

14 Méndez JCG. CONOCIMIENTOS, ACTITUDES Y PRÁCTICAS DEL. In Méndez JCG. CONOCIMIENTOS, ACTITUDES Y PRÁCTICAS DEL.; 2015. p. 70.

15 Soliz F, Sandoval ME, Reyes C, Yepez A. Medidas de Afrontamiento y Recomendaciones. Guia. . Quito: Universidad Andina Simon Bolivar, Docencia e investigacion ; 2020.

16 comercio E. Covid-19, una mayor amenaza para la mujer. El Comercio. 2020 abril.

17 G FC. TEORIAS Y MODELOS DE ENFERMERIA. [Online]. [cited 202008 13. Available from: http://artemisa.unicauca.edu.co/ pivalencia/archivos/TeoriasYModelosDeEnfermeriaYSuAplicacion. pdf.

18 Popper C. Slide share. [Online]. [cited 202008 13. Available from: https://es.slideshare.net/ceciliasp/teora-del-autociodado-dorothea-orem.

19 [Online]. [cited 202008 13. Available from: https://laestenosisaortica.wordpress.com/teorias-dedorotea-orem-autocuidado/. 